\title{
Spare Optimization Based on Renewal Process
}

\author{
Xiaoli Zou \\ School of Civil Engineering and Architecture \\ Wuhan Polytechnic University \\ Wuhan, P. R. China \\ E-mail:xiaolizou@eyou.com
}

\begin{abstract}
An optimal model for determining minimum-cost spare number for systems with components connected in series where the failure time of components follow any general distribution is presented. The component availability with a certain number of spares is derived through renewal process theory and the system availability is obtained with system reliability analysis method. The object of the optimization problem is to minimize the total spare cost of the system under the requirement of specified system availability. Genetic algorithms are used to find the optimal amount of spares for each component. Finally, an illustrative example is given.
\end{abstract}

Keywords-renewal process; system availability; spare optimization; genetic algorithms

\section{INTRODUCTION}

Predicting the optimal number of spares is one of the major concerns for many industrial organizations, especially in defense and aircraft industries because of the huge budget spent on spares every year. Spare provision plays a crucial role to ensure specified availability for a system. Theoretically it is possible to achieve high availability by including more spares. However more spares mean additional resources such as cost, space, etc. In many cases, spares are overstocked, resulting in a huge loss in revenue. For electronic and computer hardware industries, overstocked spares may not be useful because of changing technology. Thus spares required for any system should be carefully determined and if possible optimized.

Much research has been carried out on spare optimization for the system subjected to some constraints. Dynamic programming and non-linear integer programming are the two main tools used for optimizing spares. Messinger and Shooman proposed three optimization models based on dynamic programming, incremental reliability per pound and Lagrange multiplier method[1]. Sharma and Misra proposed an optimization model for repairable systems to maximize the availability of the systems[2]. The paper also presents a search technique to find the optimal solution. Kumar and Knezevic used the branch and bound procedure to solve the spare optimization problem[3]. Sarker and Haque developed a simulation model to optimize spare provisioning for the system operating with block replacement policy[4]. The main drawback of the spare optimization models studied so far is that they use special purpose algorithms to solve the optimization problems which are computationally complex. Dynamic programming becomes computationally intractable whenever the number of components is high or the number of constraints is more than one. Most of the nonlinear integer programming models are solved using approximate methods to find near optimal solution. In this paper, genetic algorithms (GAs) are applied to find the number of spares to minimize the total spare cost of the system required to achieve specified availability. GAs are search techniques based on the mechanism of natural genetics and natural selections. They make use of the artificial survival of the fittest concept with generic operators taken from nature to constitute a strong search mechanism. Moreover, GAs require neither the differential information nor the continuity involved in the objective function of an optimization problem.

The following assumptions are made about the system:

1) The failure time of the component is random and follows an arbitrary distribution.

2) The failure times of the components are statistically independent.

3) A spare component does not fail during storage.

4) The replacement time is negligible.

\section{SPARE OPTIMIZATION MODEL}

Consider a non-repairable system with $m$ components connected in series. The failure time of the component follows any general distribution like exponential, gamma, normal and Weibull. Whenever a component fails it is replaced immediately with a new identical spare if it is available. Let $X_{k}(k=1,2, \cdots)$ represents the lifetime of the $k$ th spare. Define a sequence $S_{n}$ as the follows:

$S_{n}=S_{n-1}+X_{n}$ with $S_{0}=0$

Then the sequence $\left\{S_{n} ; n=1,2, \cdots\right\}$ forms a renewal process. Let $F_{i}(t)$ be the cumulative distribution function of failure time of component ${ }^{i}$. If $n_{i}$ spares are selected for component $i$, using renewal process theory the availability of component $i$ at time ${ }^{t}$ is given by:

$$
A_{i}(t)=\sum_{k=0}^{n_{i}}\left[F_{i}^{k}(t)-F_{i}^{k+1}(t)\right]=1-F_{i}^{n_{i}+1}(t)
$$

where $F_{i}^{k}(t)$ is the $k$-fold convolution of $F_{i}(t)$. So the availability of the series system with $m$ components at time ${ }^{t}$ is given by:

$$
A_{S}(t)=\prod_{i=1}^{m} A_{i}(t)
$$


Assume $c_{i}$ is the cost of one spare for component $i$ and $A_{\min }$ is the specified system availability requirement. Now the optimization problem can be written as:

$$
\text { Min Cost }=\sum_{i=1}^{m} n_{i} \times c_{i}
$$

Subject to the following availability constraint:

$$
A_{S}(t)=\prod_{i=1}^{m} A_{i}(t) \geq A_{\text {min }}
$$

This is a non-linear integer optimization problem with one constrain. In the next section, it is suggested that genetic algorithms that can be applied successfully to find the optimal spare allocation.

\section{ApPlication of Genetic Algorithms (GAs)}

GAs belong to evolutionary algorithms and are devoted to simulate animals reproduction by Darwinian theory which stipulates that only individuals that are strong may survive and will be able to reproduce. There are many papers that give details on this family of algorithm. The general schema of GAs may be summed up as follows (Fig .1). First of all, an initial population (generation) of potential solutions (individuals) is generated randomly. A selection procedure based on a fitness function enables to choose the individuals candidate for reproduction. The reproduction consists in recombining two individuals by the crossover operator, possibly followed by a mutation of the offspring. Therefore, from the initial population a new generation is obtained. From this new generation, a second new generation is produced by the same process and so on. The stop criterion is generally based on the number of generations.

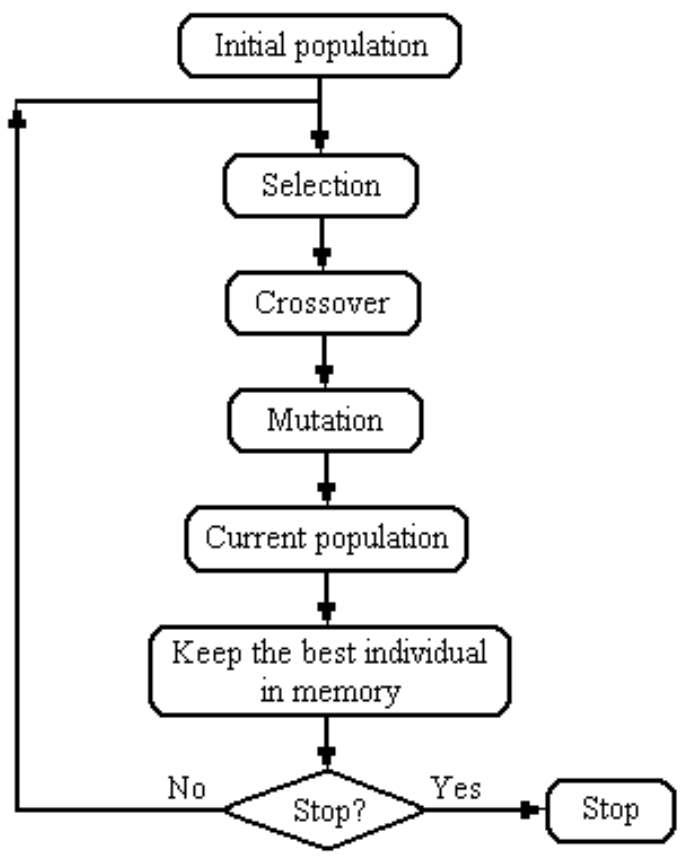

Figure 1. Flowchart representing the GAs principle

In this paper, binary codes are used for the number of each component spares. Individuals in a population are finite length strings formed from either 1 or 0 characters. Individuals and the characters are called chromosomes and artificial genes, respectively. Since the maximum number of spares for each component can be easily found from the constraint equation, the length of the individual string can be determined. Then all the individuals in the initial population are generated by randomly allocating genes into chromosomes.

The selection principle is based on Darwinian theory which postulates that only strong individuals will survive. Thus, the fitness of each individual of the population is evaluated in order to select the individual with high fitness. The availability constraint in our problem are relaxed by the penalty function technique. The express of the individual fitness is given by:

$$
\text { fitness }=\frac{C P^{2}}{\operatorname{Cost}}
$$

where

$$
C P= \begin{cases}1 & A_{S}(t) \geq A_{\text {min }} \\ \frac{A_{S}(t)}{A_{\min }} & A_{S}(t)<A_{\text {min }}\end{cases}
$$

The mainly known selection methods are the roulette wheel selection and the tournament selection. In this paper, the roulette wheel selection method is used to select the individuals for reproduction. The characteristic of the roulette wheel selection is stochastic sampling. The survival probability of each individual is determined by its fitness. A roulette wheel model is established to represent the survival probabilities for all the individuals in the population. Then the roulette wheel is rotated for several times. The number of rotating times is equal to the population size. Every rotation picks up one individual for reproduction.

The crossover operator satisfies that individuals from the mating pool recombine genetic information to generate new solutions to the problem. There are several crossover operator existing in the literature. The most current operators are the arithmetic crossover and the crossover with one or several crossing points. The one point crossover operator is used in our approach. The method of crossover with one point is as follows. Let $\left[a_{1} a_{2} \ldots a_{n}\right]$ and $\left[b_{1} b_{2} \ldots b_{n}\right]$ be two strings encoding two potential solutions to a problem. Choose randomly a value $k$ in the set $\{1, \ldots, n\}$ following an uniform law. The crossover with one crossing point consists in creating two offspring as follows: $a_{1} a_{2} \ldots a_{k} b_{k+1} b_{n}$ and $b_{1} b_{2} \ldots b_{k} a_{k+1} a_{n}$.

The mutation operator preserves diversification in the search. This operator is applied to each offspring in the population with a predetermined probability. The operator flips the gene of an offspring from 1 to 0 and vice versa at random position.

The entire processes including calculation of fitness value, selection, crossover and mutation are executed until the number of generations has reached predefined value. The final solution to the optimization problem is the 
individual with the lowest cost in all the generated individuals that satisfy the availability constraints.

\section{EXAMPLE}

Consider a four-unit series system. The time to failure distributions, cost for each spare of the component are as given in Table 1. Let availability constraint $A_{\min }=0.95$ and time $t=250$. Genetic Algorithms are used to solve this optimization problem. The population size, maximum number of generations, crossover and mutation probability are selected as $30,100,0.7$ and 0.03 , respectively.

TABLE I. SERIES SYSTEM WITH FOUR COMPONENTS

\begin{tabular}{|c|c|l|c|}
\hline No. & Distribution & Parameters & Cost \\
\hline 1 & Exponential & $\lambda=0.002$ & 250 \\
\hline 2 & Exponential & $\lambda=0.001$ & 150 \\
\hline 3 & Normal & $\mu=200 \sigma=50$ & 200 \\
\hline 4 & Normal & $\mu=230 \sigma=60$ & 400 \\
\hline
\end{tabular}

The optimal solution is given by $\left\{n_{1}=2, n_{2}=2, n_{3}=1, n_{4}=1\right\}$. That is, two spares are allocated for component 1 and 2, one spare for component 3 and 4 . The corresponding total spare cost is 1400 . Since the example concerns a simple system with few components, the computational results can be easily validated using other algorithms.

\section{CONCLUSION}

Spares play a crucial role in system life cycle cost. Though lot of research in spare optimization modeling, most of the work is based on unrealistic assumptions such as constant failure rate. The assumption of a constant failure rate provides a flexible mathematical model. However, it is not suitable for many actual systems. In this paper, the spares optimization models are developed under the assumption that the failure times of the components follow exponential, gamma, normal and Weibull distribution. The object is to minimize the total spare cost of the system satisfying a constraint on minimum required availability. Genetic Algorithms are used to solve optimization problems. The constraints are relaxed by mean of the penalty technique. A simple numerical example is presented to illustrate the effectiveness of the approach, which can be easily applied to handle systems with large components and more constraints. The models presented in the paper are useful to logistic support managers for predicting the accurate number of spares required to achieve a specified availability at a stated time. The spare optimization problem for systems with components connected in series-parallel structure can also be solved with the method in this paper. In the future, the models should be able to consider repairable systems.

\section{REFERENCES}

[1] M. Messinger and M. L. Shooman, "Techniques for optimal spares allocation - a tutorial review," IEEE Transactions on Reliability, Vol. 19, No. 4, pp. 156-166, 1970.

[2] U. Sharma and K. B. Misra, "Optimal availability design of a maintained system," Reliability Engineering and System Safety, Vol. 20, No. 2, pp. 147-159, 1988.

[3] U. D. Kumar and J. Knezevic, "Spare optimization models for series and parallel structures," Journal of Quality in Maintenance Engineering, Vol. 3, No. 3, pp. 177-188, 1997.

[4] R. Sarker and A. Haque, "Optimization of maintenance and spare provisioning policy using simulation," Applied Mathematical Modelling, Vol. 24, No. 10, pp. 751-760, 2000.

[5] E. Charles and A. Kondo, "Availability allocation to repairable systems with genetic algorithms: a multi-objective formulation," Reliability Engineering and System Safety, Vol. 82, No. 3, pp.319-330, 2003.

[6] M. S. Hayalioglu, "Optimum load and resistance factor design of steel space frames using genetic algorithm, "Structural and Multidisciplinary Optimization, Vol.21, pp. 292-299, 2001.

[7] U. D. Kumar and J. Knezevic, "Availability based spare optimization using renewal process," Reliability Engineering and System Safety, Vol. 59, No. 2, pp. 217-223, 1998. 